\title{
Séries temporais (2002-2011) de Posicionamento por Ponto GNSS, do comportamento da lonosfera e da Atividade Solar
}

\author{
Carolina Collischonn, UFRGS \\ Marcelo Tomio Matsuoka, UFRGS \\ Elen Marten de Lima, UFRGS
}

\section{Copyright 2014, SBGf - Sociedade Brasileira de Geofísica}

Este texto foi preparado para a apresentação no VI Simpósio Brasileiro de Geofísica, Porto Alegre, 14 a 16 de outubro de 2014. Seu conteúdo foi revisado pelo Comitê Técnico do VI SimBGf, mas não necessariamente representa a opinião da SBGf ou de seus associados. É proibida a reprodução total ou parcial deste material para propósitos comerciais sem prévia autorização da SBGf.

\section{Resumo}

Neste trabalho é analisada a variação do erro no posicionamento por ponto GNSS e a atividade ionosférica na região brasileira, bem como a atividade solar de janeiro/2002 a dezembro/2011, contemplando período de alta e baixa atividade solar. Para o estudo do comportamento da variação do posicionamento GNSS foram utilizados dados das estações da RBMC (Rede Brasileira de Monitoramento Contínuo) do IBGE (Instituto Brasileiro de Geografia e Estatística). Para o estudo da ionosfera foram utilizados valores de VTEC disponibilizados pelo IGS (International GNSS Service). Para o estudo da atividade solar foram utilizados os índices solares: fluxo solar no comprimento de onda de $10,7 \mathrm{~cm}$ (F10.7) e número de manchas solares (SSN).

\section{Introdução}

Uma das maiores fontes de erro sistemático nas observáveis GNSS (Global Navigation Satellite System) é a ionosfera. O erro associado à ionosfera é diretamente proporcional ao conteúdo total de elétrons livres (TEC Total Electron Content) presente ao longo do caminho da trajetória percorrida pelo sinal na ionosfera e inversamente proporcional ao quadrado da frequência do sinal. O TEC varia no tempo e no espaço e é influenciado por diversas variáveis, tais como: ciclo solar, época do ano, hora do dia, localização geográfica, atividade geomagnética, entre outros. Quanto à variação geográfica, deve-se enfatizar que o Brasil é uma das regiões do globo terrestre que possuem os maiores valores e variações espaciais e temporais do TEC (KOMJATHY et al., 2003), e desta forma, a ionosfera é especialmente uma importante fonte de erro no posicionamento com GNSS nesta região.

Os receptores GNSS de dupla freqüência (receptores geodésicos) permitem eliminar o efeito de primeira ordem da ionosfera (que corresponde a quase totalidade do erro), devido ao fato do mesmo ser dependente da freqüência do sinal. Contudo, quando são utilizados receptores GNSS de uma freqüência, não é possível utilizar essa estratégia, e os resultados obtidos sofrem a influência da ionosfera, principalmente no posicionamento por ponto e no posicionamento relativo de linhas-base médias e longas. O principal processo de formação de elétrons livres na ionosfera é a fotoionização, que consiste na absorção de radiação solar - predominantemente na faixa do extremo ultravioleta e raios-X - por elementos atmosféricos neutros (KIRCHHOFF, 1991). Dessa forma, a atividade ionosférica é extremamente influenciada pelo comportamento da atividade solar.

Durante o dia, devido ao aumento da taxa de fotoionização com o aumento da radiação solar, máximos de TEC são observados na ionosfera. Ao longo do ano, devido principalmente à mudança do ângulo zenital do Sol e da intensidade do fluxo de ionização, também são observadas variações sazonais na densidade de elétrons na ionosfera. Além da variação diária e sazonal da densidade de elétrons livres na ionosfera há ainda as variações de longo período devido ao ciclo solar de aproximadamente 11 anos.

Essas variações do ciclo solar estão associadas ao número de manchas solares, que são regiões mais escuras e frias da fotosfera solar (HATHAWAY, 2008) com campos magnéticos extremamente fortes; essas regiões são rodeadas por áreas mais brilhantes, que emitem um nível mais alto de radiação ultravioleta desencadeando, com isso, uma mudança na densidade de elétrons na ionosfera (MATSUOKA, 2007). Assim, o aumento do TEC é proporcional ao aumento do número de manchas solares.

Neste trabalho é analisada a variação do erro no posicionamento por ponto GNSS, e a atividade ionosférica na região brasileira, bem como a atividade solar de janeiro/2002 a dezembro/2011, contemplando período de alta e baixa atividade solar

\section{Metodologia}

Para o estudo do comportamento da variação do posicionamento por ponto com GNSS na região brasileira foram utilizados os dados das estações IMPZ, PARA/UFPR e POAL da RBMC (Rede Brasileira de Monitoramento Contínuo) para o período de janeiro/2002 a dezembro/2011. Estas estações foram escolhidas para contemplar diversas realidades da ionosfera no território brasileiro, com estações próximas ao equador geomagnético (IMPZ), da crista sul da anomalia equatorial (PARA/UFPR) e mais afastadas dessa crista sul (POAL).

Os dados GNSS diários destas estações foram processados no serviço de posicionamento on-line de Posicionamento por Ponto Preciso (PPP) do GSD/NRCan. Nesse processamento foram utilizadas as pseudodistâncias advindas do código $\mathrm{C} / \mathrm{A}$ na portadora L1, efemérides precisas e correções precisas dos 
relógios dos satélites. Nenhum modelo para correção do erro devido à ionosfera foi utilizado.

A partir dos resultados de erros diários nas componentes planimétrica e vertical foram gerados gráficos dos erros mensais do posicionamento de janeiro de 2002 a dezembro de 2011. Também foram geradas séries temporais (médias mensais) de 2002 a 2011 dos índices solares: fluxo solar no comprimento de onda de $10,7 \mathrm{~cm}$ (F10.7) e números de manchas solares (SSN), cujos valores foram obtidos nos seguintes endereços eletrônicos, respectivamente: http://www.ngdc.noaa.gov/nndc/struts/results?t=102827\& $\mathrm{s}=1 \& \mathrm{~d}=8,4,9$ e http://sidc.oma.be/sunspot-data/. Para o estudo do comportamento do TEC foram utilizados os arquivos no formato IONEX, disponibilizados pelo IGS (International GNSS Service), que contêm valores diários de VTEC em uma grade que cobre o globo terrestre (mapas globais da ionosfera - GIM - Global lonospheric Map).

Desses arquivos extraiu-se apenas a grade de VTEC referente à região brasileira. Após, geraram-se grades médias horárias mensais dos valores de VTEC e extraiuse dessas os valores de VTEC referentes a aproximadamente a posição de cada estação GNSS envolvida no estudo. Após, com os valores de VTEC extraídos das grades para cada estação, geraram-se gráficos representando as séries temporais de valores médios mensais de VTEC para a posição referente às estações.

\section{Resultados}

$\mathrm{Na}$ figura 1 são apresentadas as séries temporais com valores médios mensais de número de manchas solares (SSN) e fluxo solar no comprimento de onda de $10,7 \mathrm{~cm}$ (F10.7) do período de 2002 a 2011.

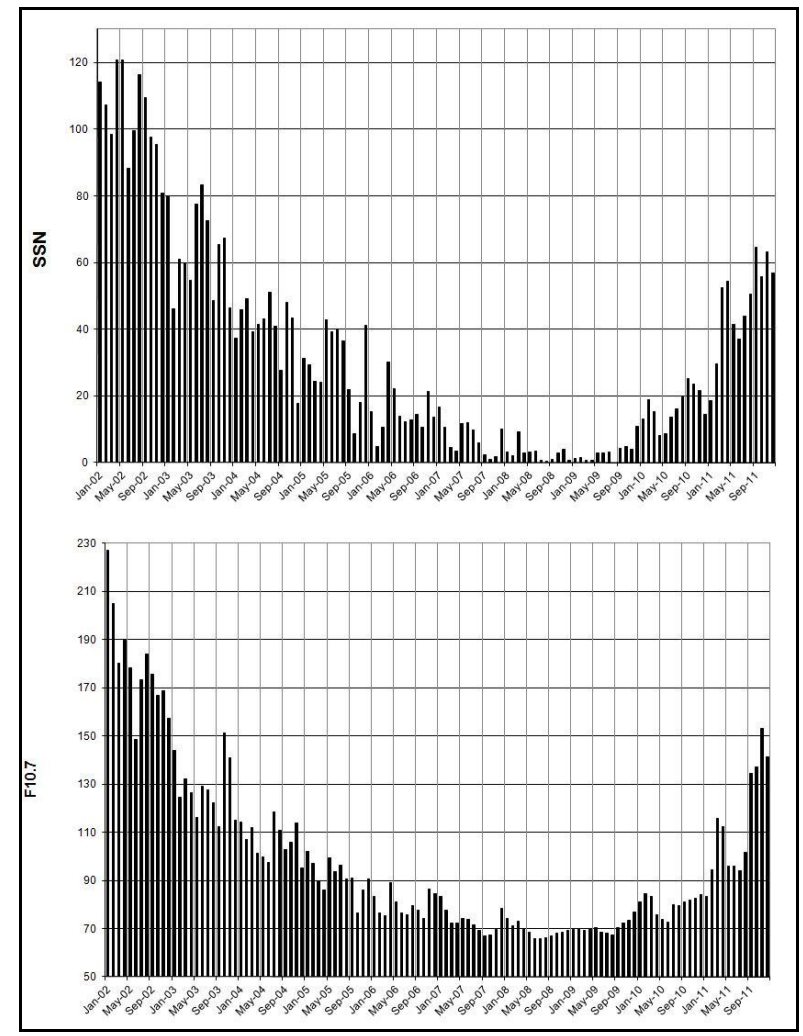

Figura 1 - Valores médios mensais dos índices solares SSN e F10.7- janeiro/2002 a dezembro/2012

Analisando a figura 1 verifica-se que há correspondência com o ciclo solar de longo período, sendo que nos anos de alta atividade solar (2002) é que se observam os maiores valores para cada índice. Após 2002 pode-se verificar uma diminuição desses valores, caracterizando o declínio para um período de baixa atividade solar. A figura 2 apresenta os valores médios mensais de VTEC para cada uma das estações GNSS. 


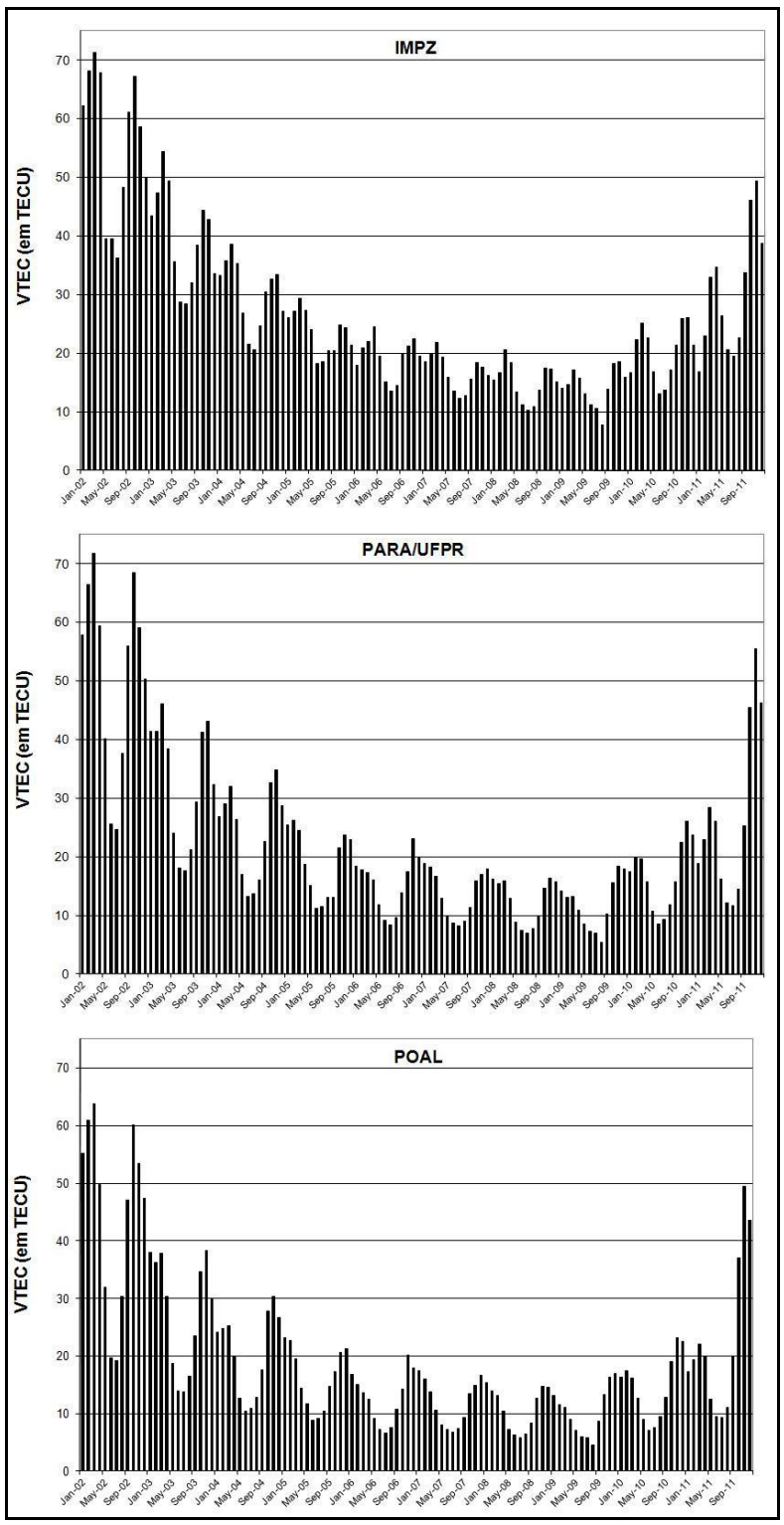

Figura 2 - Valores médios mensais de VTEC para as estações IMPZ, PARA/UFPR e POAL - janeiro/2002 a dezembro/2012.

Analisando a figura 2 verifica-se a variação semi-anual do VTEC e a de longo período. Com relação à variação semi-anual constatam-se valores mais altos de VTEC nos meses próximos aos equinócios e solstício de verão, e menores nos meses de inverno. Além disso, em anos em período de alta atividade solar, os meses mais próximos aos equinócios apresentam valores maiores que nos próximos ao solstício de verão.

Verifica-se claramente também a variação de longo período. Além disso, a variação semi-anual em anos de alta atividade solar é maior do que a verificada em anos de baixa atividade solar. Nota-se que a diferença anual entre os valores máximos e mínimos para a estação IMPZ (mais próxima do equador geomagnético) é menor do que a diferença das outras estações (PARA/UFPR e POAL).

As séries temporais dos erros médios mensais na componente vertical (Ev) para as estações IMPZ, PARA/UFPR e POAL de janeiro de 2002 a dezembro de 2011 são apresentados na figura 3 . Analisando a figura 3 pode-se observar o comportamento semi-anual do erro vertical, com maiores valores nos meses de equinócios e verão, e menores valores nos meses de inverno. Essa variação semi-anual é maior nos anos de alta atividade solar. 


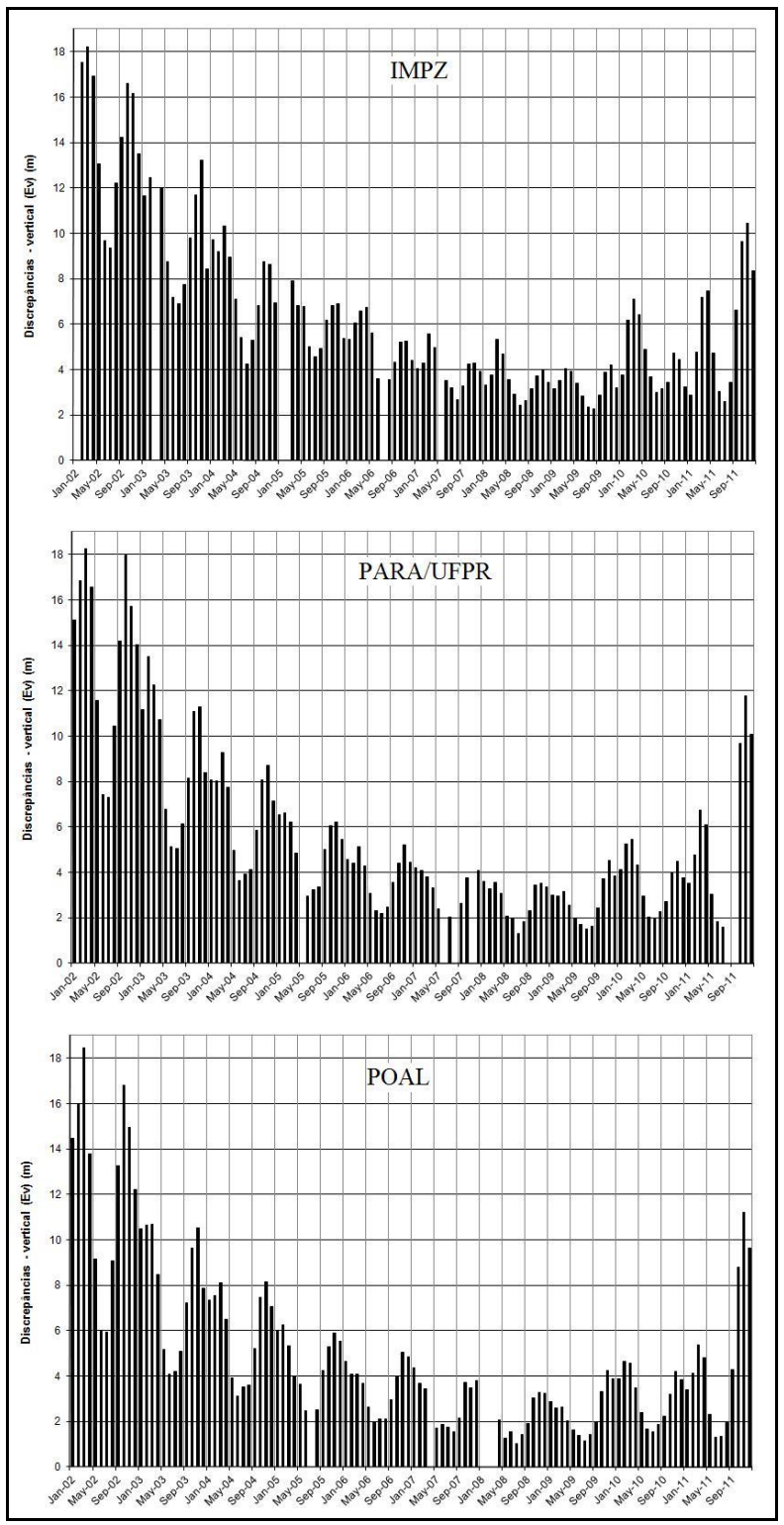

Figura 3 - Valores médios mensais de erro vertical para as estações IMPZ, PARA/UFPR e POAL - janeiro/2002 a dezembro/2011.

A figura 4 apresenta os valores médios mensais para o erro em planimetria (Ep) de janeiro de 2002 a dezembro de 2011. Analisando a figura 4 e comparando com a figura 3 , verifica-se que 0 erro médio mensal em planimetria é menor do que o erro vertical. O que era esperado, pois a geometria (distribuição) dos satélites favorece a determinação planimétrica, já que parte do erro devido à ionosfera que contamina as observáveis GNSS é cancelada no plano horizontal.

Ainda pode-se verificar na figura 4 que os erros em planimetria para a estação IMPZ são menores do que os referentes às outras duas estações. A estação IMPZ está próxima ao equador e a geometria dos satélites favorece ainda mais a compensação dos erros no plano horizontal.
Isto porque a distribuição dos satélites é mais homogênea em termos azimutais para uma estação próxima ao equador.

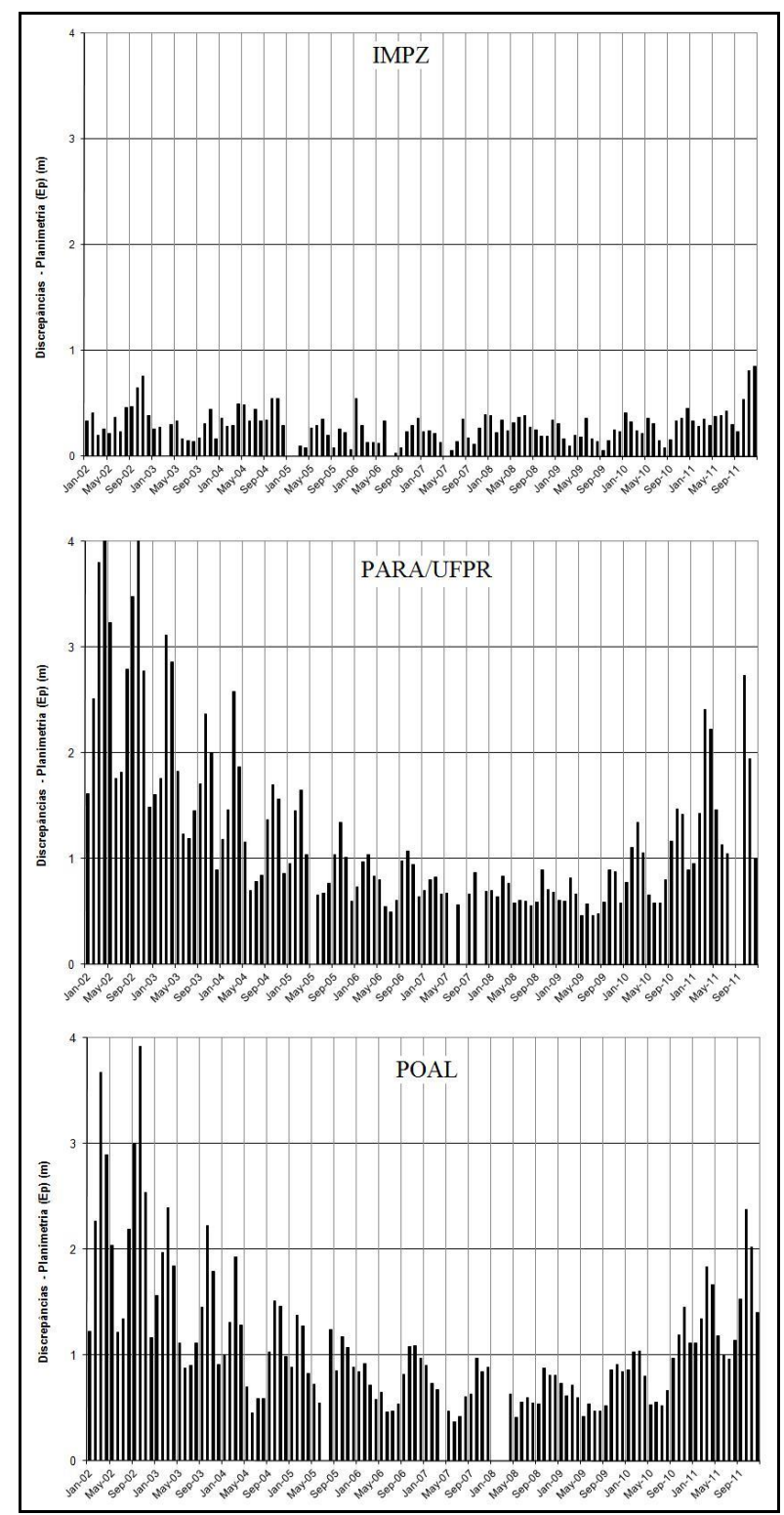

Figura 4 - Valores médios mensais de erro em planimetria para as estações IMPZ, PARA/UFPR e POAL - janeiro/2002 a dezembro/2011.

\section{Discussão e Conclusões}

Nesse artigo foi realizada a análise da variação do posicionamento por ponto GNSS em diferentes locais da região brasileira, bem como do comportamento da ionosfera (VTEC) e da atividade solar (índices solares SSN e F10.7). A partir da análise das series temporais de índices solares se verifica correspondência com o ciclo solar de longo período, bem como na análise das séries 
temporais de valores de VTEC para as estações IMPZ, PARA/UFPR e POAL. Nas series temporais de VTEC também se verifica a variação semi-anual, que em anos de alta atividade solar é maior do que a verificada em anos de baixa atividade solar. As séries temporais do posicionamento por ponto GNSS mostram os erros médios mensais na componente vertical e planimétrica para as estações IMPZ, PARA/UFPR e POAL. A partir da análise das séries temporais para o posicionamento na componente vertical pode-se observar o comportamento semi-anual do erro, com maiores valores nos meses de equinócios e verão, e menores valores nos meses de inverno. Essa variação semi-anual é maior nos anos de alta atividade solar. Pode-se verificar também que a variação dos valores médios mensais do erro vertical para a estação IMPZ é menor do que a variação para as outras duas estações. Isso se deve a localização da estação IMPZ, próxima ao equador geomagnético. A partir da análise das séries temporais para 0 posicionamento também se verifica que o erro médio mensal em planimetria é menor que o erro vertical, o que concorda com o esperado, pois a geometria (distribuição) dos satélites favorece a determinação planimétrica e parte do erro devido à ionosfera que contamina as observáveis GNSS é cancelada no plano horizontal. Também é observado que os erros em planimetria para a estação IMPZ são menores do que os referentes às outras duas estações.

\section{Agradecimentos}

Ao CNPq (Universal/Proc.481339/2010-8; Bolsa Produtividade em Pesquisa Proc.307472/2009-4 e; Bolsas IC PIBIC/UFRGS/2010-2011 e 2011-2012 e Bolsa de mestrado 2013-2014). À Propesq/UFRGS pelas Bolsas BIC/2008-2009 e 2009-2010. À Fapergs (ARD/003/2009 Proc. 0901842). Ao GSD/NRCan pelo serviço online de PPP. Ao IBGE pelos dados da RBMC.

\section{Referências}

HATHAWAY, D. H.. The Sunspot Cycle. 2008. Disponível em:

http://solarscience.msfc.nasa.gov/sunspotcycle.shtml.

Acesso em: Junho, 2008.

KIRCHHOFF, V.W.J.H.. Introdução à geofísica espacial. São Paulo: Nova Stella, Ed. USP/FAPESP, 1991. 149p.

KOMJATHY, A.; SPARKS, L.; MANNUCCI, A.J.; XIAOQING, P.. An assessment of the current WAAS ionospheric correction algorithm in the south American region. Journal of the Institute of Navigation, v.50, n.3, p.193-218, Fall/2003.
MATSUOKA, M.T.. Influência de diferentes condições da ionosfera no posicionamento por ponto com GPS: Avaliação na região brasileira. 2007. 263p. Tese (Doutorado em Ciências Cartográficas) - Programa de Pós-Graduação em Ciências Cartográficas, Faculdade de Ciências e Tecnologia, Universidade Estadual Paulista, Presidente Prudente. 